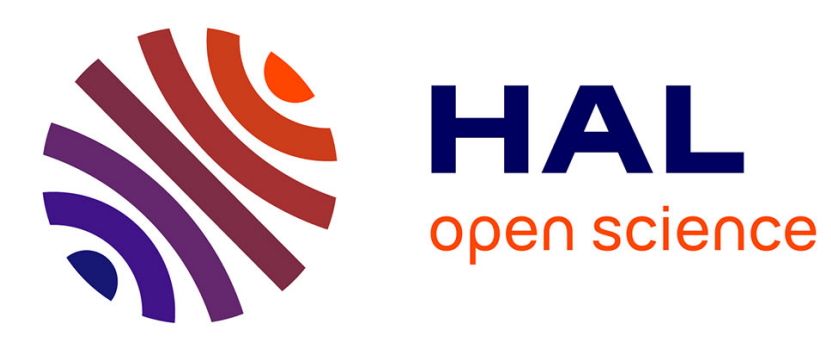

\title{
Feasibility of Metal Pad Roll Instability Experiments at Room Temperature
}

\author{
Caroline Nore, L. Cappanera, J.-L. Guermond, T. Weier, W. Herreman
}

\section{To cite this version:}

Caroline Nore, L. Cappanera, J.-L. Guermond, T. Weier, W. Herreman. Feasibility of Metal Pad Roll Instability Experiments at Room Temperature. Physical Review Letters, 2021, 126 (18), 10.1103/PhysRevLett.126.184501 . hal-03335970

\section{HAL Id: hal-03335970 https://hal.science/hal-03335970}

Submitted on 6 Sep 2021

HAL is a multi-disciplinary open access archive for the deposit and dissemination of scientific research documents, whether they are published or not. The documents may come from teaching and research institutions in France or abroad, or from public or private research centers.
L'archive ouverte pluridisciplinaire HAL, est destinée au dépôt et à la diffusion de documents scientifiques de niveau recherche, publiés ou non, émanant des établissements d'enseignement et de recherche français ou étrangers, des laboratoires publics ou privés. 


\title{
Feasibility of metal pad roll instability experiments at room temperature
}

\author{
C. Nore ${ }^{1}$ L. Cappanera, ${ }^{2}$ J.-L. Guermond, ${ }^{3}$ T. Weier, ${ }^{4}$ and W. Herreman ${ }^{1}$ \\ ${ }^{1}$ Université Paris-Saclay, CNRS, LIMSI, 91400 Orsay, France \\ ${ }^{2}$ Department of Mathematics, University of Houston, Houston, TX 77204-3008, USA \\ ${ }^{3}$ Department of Mathematics, Texas AEM University 3368 TAMU, College Station, TX 77843-3368, USA \\ ${ }^{4}$ Helmholtz-Zentrum Dresden-Rossendorf, Germany
}

(Dated: March 26, 2021)

\begin{abstract}
Combining theoretical arguments and numerical simulations, we demonstrate that the metal pad roll instability can occur in a centimeter scale set-up with reasonable values of the magnetic field and electrical current and with metal pairs that are liquid at room temperature. We investigate two fluid pairs: gallium with mercury (immiscible pair) or gallium with GaInSn eutectic alloy (miscible pair).
\end{abstract}

PACS numbers:

Introduction Since 1886, aluminium is produced in Hall-Héroult reduction cells by driving very large electrical currents through two shallow fluid layers: the top layer is a mixture of cryolite and alumina and the bottom layer is composed of liquid aluminium. Ideally, both fluid layers should be at rest, but gravity waves may spontaneously grow at the interface of the two liquids when a weak ambient magnetic field is present. This phenomenon, called metal pad roll instability [1 6 ], can cause short-circuits that degrade the carbon anodes faster than it should. Although the physical origin of this instability has been known for a while [2, there are very few experiments that reproduce it in less hostile environment (Hall-Héroult cells operate at around $1000^{\circ} \mathrm{C}$ ). So far, only one experiment [7] has succeeded in reproducing this instability in reasonable conditions. But since in this experiment stainless steel needles replace the top fluid layer, comparisons with theoretical fluid-based predictions are difficult. The idea to use liquid metal pairs other than cryolite and aluminium is attractive, but it is also hard to test with classical stability theories [5, 6] that implicitly assume the conductivity jump to be large. Using the recent stability theory from [8] and numerical simulations, we show in this letter that the metal pad roll instability can occur in a centimeter-scale apparatus with fairly low electrical currents and imposed magnetic fields and with pairs of metals that are liquid at room temperature. We investigate two fluid pairs: (i) gallium (top layer) with mercury (bottom layer) as an immiscible case; (ii) gallium (top layer) with eutectic GaInSn alloy (bottom layer) as a miscible case.

Set-up The idealized set-up investigated in this letter is shown in Fig. 1.(a). In a cylinder of radius $R$, a fluid layer of height $H_{1}$ and composed of liquid Ga floats on top of a fluid layer of height $H_{2}$ and composed of either $\mathrm{Hg}$ (immiscible case) or eutectic alloy $\mathrm{Ga}_{67} \mathrm{In}_{20.5} \mathrm{Sn}_{12.5}$ (in wt\%, miscible case). The cell is subjected to a uniform vertical magnetic field $\boldsymbol{B}^{e}=B_{z} \boldsymbol{e}_{z}$ and is traversed by a homogeneous current density $\boldsymbol{J}=J \boldsymbol{e}_{z}$. The Seleinstability mechanism [2] goes as follows (Fig. 1.(b) and

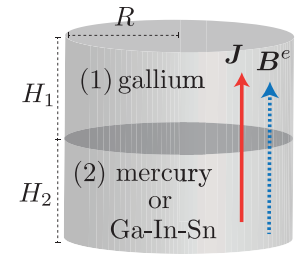

(a)

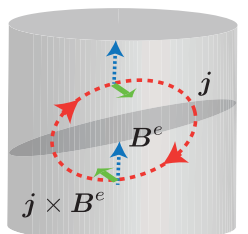

(b)

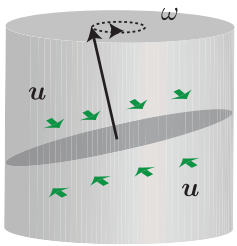

(c)
FIG. 1: Sketch of the cylindrical set-up and instability mechanism. (a) A layer of gallium floats on a layer of mercury or eutectic GaInSn alloy. A current $\boldsymbol{J}$ passes through the cell which is subjected to an external vertical magnetic field $\boldsymbol{B}^{e}$. (b) Due to the conductivity difference (gallium is a better conductor), a small excess current $\boldsymbol{j}$ appears as soon as the interface is inclined. This excess current interacts with the external magnetic field and generates a Lorentz force $\boldsymbol{j} \times \boldsymbol{B}^{e}$ (green arrows). (c) This force transfers power $\left(\left(\boldsymbol{j} \times \boldsymbol{B}^{e}\right) \cdot \boldsymbol{u}>0\right)$ to a wave that rotates anti-clockwise when seen from above.

(c)): since $\mathrm{Ga}$ is a slightly better conductor than $\mathrm{Hg}$ and the GaInSn alloy, any interface elevation reorganizes the electrical current into $\boldsymbol{J}+\boldsymbol{j}$ in order to pass preferentially through the Ga; the current excess $j$ interacts with the external magnetic field and the resulting Lorentz force $\boldsymbol{j} \times \boldsymbol{B}^{e}$ transfers power to a rotating gravity wave.

\begin{tabular}{ccccc}
\hline \hline layer & metal & $\rho\left(\mathrm{kgm}^{-3}\right)$ & $\sigma\left(\mathrm{Sm}^{-1}\right)$ & $\eta(\mathrm{mPas})$ \\
\hline 1 & $\mathrm{Ga}$ & 6077 & $3.86 \times 10^{6}$ & 1.686 \\
$2^{a}$ & $\mathrm{Hg}$ & 13524 & $1.03 \times 10^{6}$ & 1.51 \\
$2^{b}$ & $\mathrm{Ga}_{67} \mathrm{In}_{20.5} \mathrm{Sn}_{12.5}$ & 6345 & $3.24 \times 10^{6}$ & 2.05 \\
\hline \hline
\end{tabular}

TABLE I: Material properties at $T=303 \mathrm{~K}$ (from 9, 10, for Ga and 11 for GaInSn): Ga (Layer 1); Hg (Layer $2^{a}$, immiscible pair); GaInSn eutectic alloy (Layer $2^{b}$, miscible pair).

The density $\rho$, dynamical viscosity $\eta$ and electrical conductivity $\sigma$ of both metals are listed in Table I The immiscibility of the pair $\mathrm{Ga} \mathrm{\&} \mathrm{Hg}$ is interesting since experiments can be repeated many times with the same fluids. But since $\mathrm{Hg}$ is toxic and regulated, it is also interesting to investigate the miscible pair Ga \& GaInSn eutectic. The diffusion coefficient $D$ that controls the mixing of Ga 
\& GaInSn is not precisely known, but it is conjectured to be close to $1.7 \times 10^{-9} \mathrm{~m}^{2} \mathrm{~s}^{-1}$, which is the self-diffusion coefficient in liquid Ga. The diffusion time-scale $R^{2} / D$ is about $59000 \mathrm{~s}(16 \mathrm{~h})$ for $R=1 \mathrm{~cm}$. Even though this pair is miscible, the diffusion time is large enough to allow many experiments to be conducted without having to change the fluids in the device.

Theory for immiscible liquids We use the linear theory developed in [8] to assess the stability of rotating gravity waves in cylindrical cells. Using the notation of [8], we now recall the key elements of this theory. The vertical deformation of the interface about the rest state $z=0$ is shown therein to be of the form $z \approx A J_{m}(k r) e^{\mathrm{i}(m \theta+\omega t)} e^{\lambda t}$, where $A$ is the arbitrary amplitude, $J_{m}$ is a Bessel function, $m \in \mathbb{Z}$ is the azimuthal wavenumber, $k=\kappa_{m n} R^{-1}$ is the radial wavenumber, and $\kappa_{m n}$ is the $n$-th zero of $J_{m}^{\prime}$, i.e. $J_{m}^{\prime}\left(\kappa_{m n}\right)=0$. The dispersion relation is

$$
\omega= \pm \sqrt{\frac{\left(\rho_{2}-\rho_{1}\right) g k}{\rho_{1} \tanh ^{-1}\left(k H_{1}\right)+\rho_{2} \tanh ^{-1}\left(k H_{2}\right)}},
$$

where $g=9.81 \mathrm{~ms}^{-2}$. The growth rate $\lambda$ is the sum of three contributions $\lambda=\lambda_{\mathrm{v}}+\lambda_{\mathrm{vv}}+\lambda_{\mathrm{visc}}$. Assuming that $\left.j_{z}\right|_{z=H_{1}}=\left.j_{z}\right|_{z=-H_{2}}=0$ in order to mimic solid electrodes that do not conduct electricity as well as gallium, GaInSn alloy or mercury, we find the destabilizing term

$$
\lambda_{\mathrm{v}}=\frac{\omega}{2} \frac{J B_{z}}{\left(\rho_{2}-\rho_{1}\right) g} \frac{m}{(k R)^{2}-m^{2}} \Xi,
$$

with

$$
\Xi=\frac{\left(\sigma_{1}^{-1}-\sigma_{2}^{-1}\right) \sum_{i=1,2}\left[\frac{k H_{i}}{\sinh ^{2}\left(k H_{i}\right)}+\frac{1}{\tanh \left(k H_{i}\right)}\right]}{\sum_{i=1,2}\left(\sigma_{i} \tanh \left(k H_{i}\right)\right)^{-1}} .
$$

(see supplementary material of [8]). Other types of electrical boundary conditions are discussed in the supplementary material and only weakly affect the instability as the cell studied here is rather tall. With gallium (fluid 1) and either mercury or GaInSn alloy (fluid 2), we have $\left(\sigma_{1}^{-1}-\sigma_{2}^{-1}\right)<0$. If we choose $J B_{z}>0$, only waves rotating anti-clockwise $(m \omega<0)$ can, i.e. can be such that $\lambda_{\mathrm{v}}>0$. This is in agreement with Fig. 1. The magnetic damping $\lambda_{\mathrm{vv}}<0$, proportional to $B_{z}^{2}$, is calculated using a numerical approximation of [8, eq. (2.64)]. The viscous damping $\lambda_{\text {visc }}<0$ is available from [8, eq. (2.66)]. Focusing on cells with $H_{1}=H_{2}=R$ in the centimeter range, we give in Table II numerical values for $\lambda_{\mathrm{v}} / J B_{z}, \lambda_{\mathrm{vv}} / B_{z}^{2}$ and $\lambda_{\text {visc }}$ for the fundamental wave $\left(m=1, \kappa_{11}=1.841\right)$, which is always the most unstable. Smaller cells have larger $\lambda_{\mathrm{v}}$ but are also subject to more viscous damping. The magnetic damping is independent of the size of the system. Using $R=2 \mathrm{~cm}$, we show in Fig. 2 the growth rate $\lambda$ in the $J-B_{z}$ plane. The maximal current density and magnetic field used here are fairly large,

\begin{tabular}{lcccc}
\hline \hline$R(\mathrm{~cm})$ & $|\omega|\left(\mathrm{s}^{-1}\right)$ & $\lambda_{\mathrm{v}} /\left(J B_{z}\right)$ & $\lambda_{\mathrm{vv}} / B_{z}^{2}$ & $\lambda_{\text {visc }}\left(\mathrm{s}^{-1}\right)$ \\
\hline 1 & 25.55 & $1.00 \times 10^{-4}$ & -39.6 & -0.262 \\
2 & 18.06 & $7.08 \times 10^{-5}$ & -39.6 & -0.110 \\
5 & 11.42 & $4.48 \times 10^{-5}$ & -39.6 & -0.0350 \\
\hline \hline 1 & 6.09 & $10^{-4}$ & -115 & -0.18 \\
2 & 4.30 & $7.1 \times 10^{-5}$ & -115 & -0.0759 \\
5 & 2.72 & $4.48 \times 10^{-5}$ & -115 & -0.0242 \\
\hline \hline
\end{tabular}

TABLE II: Theoretical values of the growth rates of the fundamental wave $m=1, \kappa_{11}=1.841 \mathrm{in}$ cells with varying size $R=H_{1}=H_{2} \cdot \lambda_{\mathrm{v}} / J B_{z}$ in units of $\mathrm{s}^{-1} \mathrm{~m}^{2} \mathrm{~A}^{-1} \mathrm{~T}^{-1}, \lambda_{\mathrm{vv}} / B_{z}^{2}$ in units of $\mathrm{s}^{-1} \mathrm{~T}^{-2}$. Rows 2-4: Immiscible fluid pair $\mathrm{Ga} \& \mathrm{Hg}$. Rows 5-7: Miscible fluid pair Ga \& GaInSn eutectic alloy.
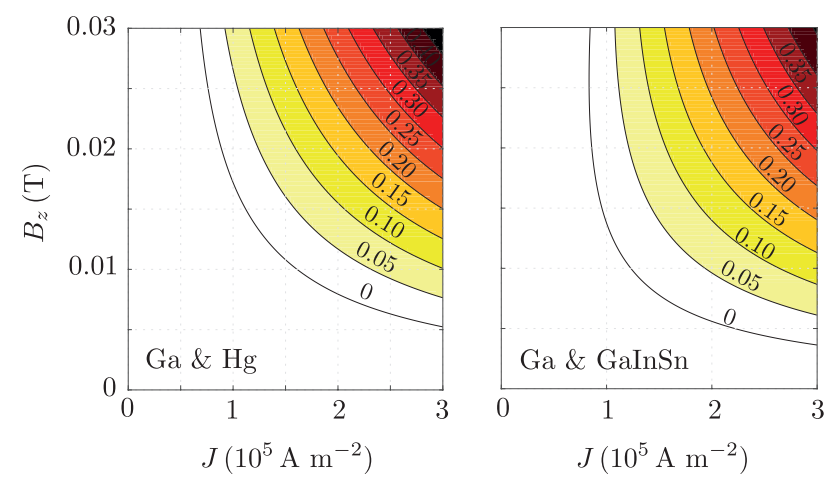

FIG. 2: Growth rate $\lambda$ in $\mathrm{s}^{-1}$ of the fundamental wave $(m=1$, $\left.\kappa_{11}=1.841\right)$ in the $J-B_{z}$ plane in a $R=H_{1}=H_{2}=2 \mathrm{~cm}$ set-up with layers of $\mathrm{Ga} \& \mathrm{Hg}$ or $\mathrm{Ga} \& \mathrm{GaInSn}$ eutectic.

$J \leq 3 \times 10^{5} \mathrm{Am}^{-2}$ and $B_{z} \leq 30 \mathrm{mT}$, but these values are definitely accessible. This suggests that the metal pad roll instability can be observed in realistic set-ups. In Fig. 3 we fix $B_{z}=15 \mathrm{mT}$ and show the theoretical growth rate as a function of $J$ and $I=J \pi R^{2}$ for both set-ups.

Numerical simulations We now confront the theory to numerical simulations done with SFEMaNS [12, 13]. This code combines spectral and finite-element techniques and is designed to solve various magnetohydrodynamical problems. We focus on the cell with geometry $R=H_{1}=H_{2}=2 \mathrm{~cm}$. We fix $B_{z}=15 \mathrm{mT}$ and vary $J$.

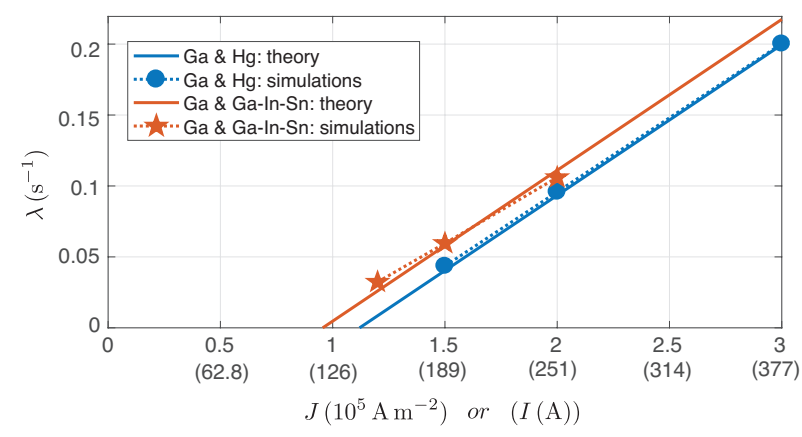

FIG. 3: Growth rate $\lambda$ in $\mathrm{s}^{-1}$ of the fundamental wave $(m=1$, $\left.\kappa_{11}=1.841\right)$ as a function of $J$ or $I$ in a $R=H_{1}=H_{2}=2 \mathrm{~cm}$ set-up with magnetic field $B_{z}=15 \mathrm{mT}$ 
We start by simulating the metal pad roll instability in the immiscible $\mathrm{Ga} \& \mathrm{Hg}$ set-up. We use a multiphase method similar to that of $[\underline{8}, 14$. The main novelty though is that the incompressibility is now enforced via an artificial compressibility method which allows for a better control on the divergence of the velocity. A short description of the method is provided in the supplementary material. We initialize the calculation with a small amplitude rotating gravity wave $(m, n)=(1,1)$. To capture correctly the viscous damping, we need to resolve spatial scales as small as the thickness of the viscous boundary layer, $\sqrt{\nu_{2} / \omega}=0.08 \mathrm{~mm}$. The meridian finite element grids are locally refined at this scale near the solid boundary and the fluid interface. The timesteps are of the order $10^{-4} s$, and we use 32 real Fourier modes in the azimuthal direction. A convergence study is reported in the supplementary material. Three simulations are done with $J \in(1.5,2,3) \times 10^{5} \mathrm{Am}^{-2}$. We observe in each case a positive growth rate and the wave rotates anti-clockwise as expected (see Fig. 1 and video in the supplementary material). Fig. 4. (a) shows time-
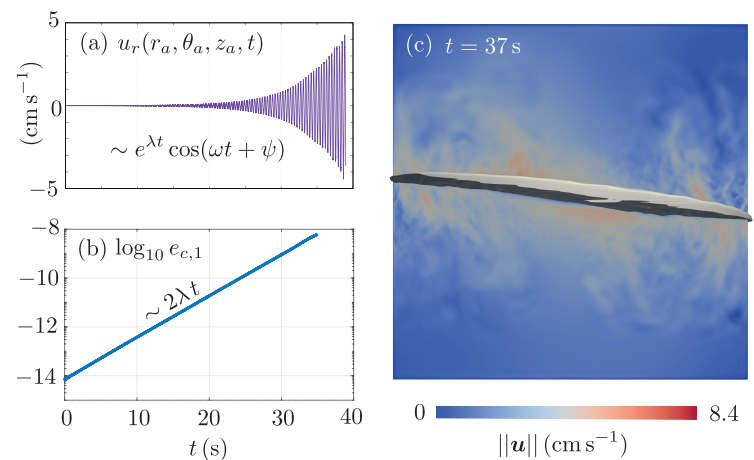

FIG. 4: Simulations in the immiscible Ga \& Hg set-up. (a) Time-series of the velocity at $r_{a}=0, \theta_{a}=0, z_{a}=-5 \mathrm{~mm}$ gives $\omega$. (b) Time-series of $e_{c, 1}$ gives $\lambda\left(e_{c, 1}\right.$ is the kinetic energy of the azimuthal Fourier mode $m=1$ ). (c) Iso-surface $\rho=\left(\rho_{1}+\rho_{2}\right) / 2$ and turbulent structures in the vertical plane $x=0$ at $t=37 \mathrm{~s}$.

series of the velocity at $\left(r_{a}, \theta_{a}, z_{a}\right)=(0,0,-5 \mathrm{~mm})$ from which we obtain $\omega$. We measure $\omega_{\text {num }}=17.7 \mathrm{~s}^{-1}$ for $J=3 \times 10^{5} \mathrm{Am}^{-2}$. This value is very close to the theoretical frequency $\omega_{\text {th }}=\omega+\lambda_{\text {visc }}=17.95 \mathrm{~s}^{-1}$ (with viscosity correction). Fig. 4.(b) shows time-series of the kinetic energy $e_{c, 1}$ that is carried by the azimuthal Fourier mode $m=1$. The exponential growth is unambiguous and a linear fit allows us to measure $2 \lambda$. We obtain $\lambda_{\text {num }}=(0.0436,0.09584,0.2003) \mathrm{s}^{-1}$ for $J=$ $(1.5,2,3) \times 10^{5} \mathrm{Am}^{-2}$. Fig. 3 shows that these values are in excellent agreement with our theory. Fig. 4. (c) shows a snapshot of the deformed interface and of the local flowspeed $\|\boldsymbol{u}\|$ at time $t=37 \mathrm{~s}$. Although the growth seems to be still in the linear stage (see Fig. 4.(b)), the local flow-speed is already large: $U \sim 8 \mathrm{cms}^{-1}$. In the mercury, the Reynolds number is about $R e=\rho_{2} U R / \eta_{2}=14000$.
Transition to turbulence seems to have occurred.

Simulating the metal pad roll instability with the miscible metal pair, Ga \& GaInSn, requires a different numerical approach to allow mixing of the metals. Here we use a model similar to that of 15 . Since it is reasonable to suppose that In and Sn diffuse at roughly the same rate in $\mathrm{Ga}$, we use the cumulated mass-concentration of indium and tin in the alloy $\rho_{\text {IS }}=\rho_{\text {In }}+\rho_{\text {Sn }}$ to track the composition of the alloy. We suppose that Ga and the GaInSn eutectic are perfectly separated at time $t=0$, i.e. $\left.\rho_{\text {IS }}\right|_{t=0}=\rho_{\text {IS }}^{\text {eut }} \mathcal{H}(-z)$ where $\mathcal{H}$ is the Heaviside function and $\rho_{\text {IS }}^{\text {eut }}=(0.205+0.125) \rho_{2}=2094 \mathrm{kgm}^{-3}$ is the mass concentration of indium and tin combined in the $\mathrm{Ga}_{67} \mathrm{In}_{20.5} \mathrm{Sn}_{12.5}$ eutectic alloy (in wt \%). The velocity and the magnetic induction are denoted $\boldsymbol{u}$ and $\boldsymbol{b}$. We numerically solve the evolution equations:

$$
\begin{gathered}
\rho_{*}\left(\partial_{t} \boldsymbol{u}+(\boldsymbol{u} \cdot \boldsymbol{\nabla}) \boldsymbol{u}\right)=-\nabla p-\left(\rho_{*}+\chi \rho_{\mathrm{IS}}\right) g \boldsymbol{e}_{z} \\
\quad+\boldsymbol{\nabla} \cdot\left(\eta_{*}\left(\nabla \boldsymbol{u}+(\nabla \boldsymbol{u})^{\mathrm{T}}\right)\right)+\boldsymbol{j} \times \boldsymbol{b}, \\
\partial_{t} \boldsymbol{b}=\boldsymbol{\nabla} \times(\boldsymbol{u} \times \boldsymbol{b})-\mu_{0}^{-1} \nabla \times\left(\sigma_{*}^{-1}+\alpha \rho_{\mathrm{IS}}\right) \boldsymbol{\nabla} \times \boldsymbol{b}, \\
\boldsymbol{\nabla} \cdot \boldsymbol{u}=0, \quad \boldsymbol{\nabla} \cdot \boldsymbol{b}=0, \\
\partial_{t} \rho_{\mathrm{IS}}+\boldsymbol{u} \cdot \boldsymbol{\nabla} \rho_{\mathrm{IS}}=D \nabla^{2} \rho_{\mathrm{IS}} .
\end{gathered}
$$

Linear approximations are used for the buoyancy and the resistivity with $\chi=\left(\rho_{2}-\rho_{1}\right) / \rho_{\mathrm{IS}}^{\text {eut }}=0.128$ and $\alpha=\left(\sigma_{2}^{-1}-\sigma_{1}^{-1}\right) / \rho_{\text {IS }}^{\text {eut }}=2.368 \times 10^{-11} \Omega \mathrm{m}^{4} \mathrm{~kg}^{-1}$. The reference density and conductivity are that of pure Ga: $\rho_{*}=\rho_{1}, \sigma_{*}=\sigma_{1}$. These approximations are reasonable as the material properties vary little between pure Ga and the GaInSn eutectic. Using the dispersion relation (1) (with $\rho_{2} \tanh ^{-1}\left(k H_{2}\right) \approx \rho_{1} \tanh ^{-1}\left(k H_{2}\right)$ in the denominator) we estimate that the Boussinesq approximation alters the frequency of gravity waves by less than $1 \%$. We also use a constant dynamical viscosity $\eta_{*}=\left(\eta_{1}+\eta_{2}\right) / 2=$ $1.87 \times 10^{-3} \mathrm{Pas}$ in the alloy. According to [8, Eq. (2.66)] this affects the viscous damping rate $\lambda_{\text {visc }}$ by less than $0.5 \%$. The no-slip boundary condition and the zero flux of In and Sn across the boundaries are enforced. The boundary conditions on the magnetic field are as in 8 . We run simulations with $J=(1.25,1.5,2) \times 10^{5} \mathrm{Am}^{-2}$.
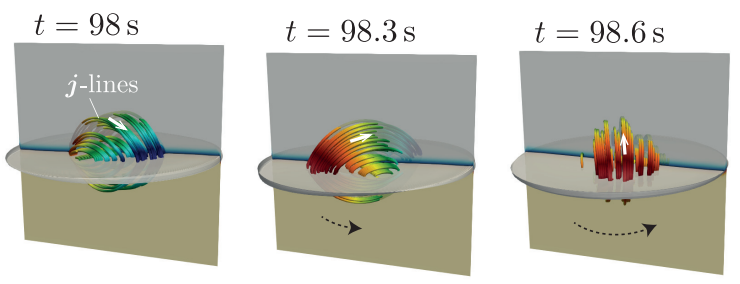

$$
\frac{\rho\left(\mathrm{kg} \mathrm{m}^{-3}\right)}{6077 \quad 6345}
$$

FIG. 5: Emergence of a rotating gravity wave in Ga \& GaInSn eutectic (miscible) set-up, $J=1.5 \times 10^{5} \mathrm{Am}^{-2}$. We show the iso-surface $\rho=\left(\rho_{1}+\rho_{2}\right) / 2$, the density $\rho$ in color code in the vertical plane $x=0$ and lines of excess current $\boldsymbol{j}$.

The fluids are initially at rest. Contrary to the immis- 
cible case, a long transient precedes the growth of the gravity wave during which the fluids start to blend by diffusion. For $J=1.5 \times 10^{5} \mathrm{Am}^{-2}$, we observe a rotating wave after $90 \mathrm{~s}$, here illustrated by 3 snapshots in Fig. 5 . For the fundamental wave the immiscible theory gives $\omega_{\text {th }}=\omega+\lambda_{\text {visc }}=4.23 \mathrm{~s}^{-1}$ with $J=1.5 \times 10^{5} \mathrm{Am}^{-2}$. Here we measure $\omega_{\text {num }}=4.22 \mathrm{~s}^{-1}$, which is in excellent agreement with the theory. The growth rates measured numerically are $\lambda_{\text {num }}=(0.0323,0.0596,0.106) \mathrm{s}^{-1}$ for $J=(1.25,1.5,2) \times 10^{5} \mathrm{Am}^{-2}$. Fig. 3 shows that these values agree well with the immiscible theory. Hence the initial phase of the instability is not affected by the miscibility. Doing long-time simulations with large waves is very costly: with $D=1.7 \times 10^{-9} \mathrm{~m}^{2} \mathrm{~s}^{-1}$ and $U=1 \mathrm{cms}^{-1}$, the Péclet number is as high as $P e=U R / D=10^{5}$. To gain some insight on the nonlinear regime of the miscible metal pad roll instability at a reasonable numerical cost, and to gain some insight in a more pessimistic scenario in which the Ga and GaInSn eutectic would blend more easily by diffusion, we now investigate what happens by using a much larger diffusivity: $D_{\text {fake }}=100 D=1.7 \times 10^{-7} \mathrm{~m}^{2} \mathrm{~s}^{-1}$. In Fig. 6, we show a snapshot at time $t=31.35 \mathrm{~s}$ of the strongly deformed interface, the excess current, and $u_{y}$ in the plane $\{x=0\}$ (video available in the supplementary material). The current density is very large $J=4 \times 10^{5} \mathrm{Am}^{-2}$, giving $U \sim 5 \mathrm{cms}^{-1}$ and the Péclet number $P e_{\text {fake }} \sim 5800$ now numerically accessible. The metal pad roll instability occurs despite the enhanced mixing.
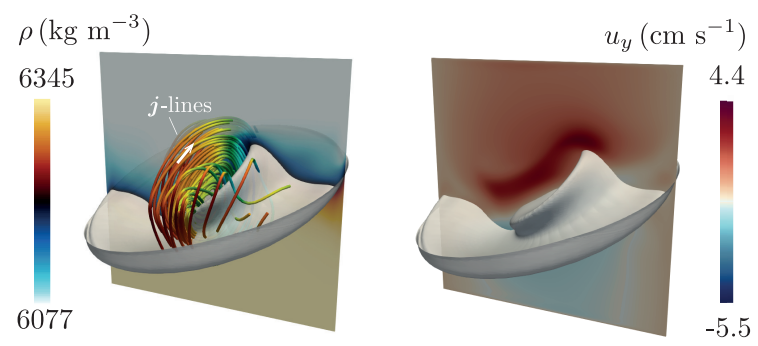

FIG. 6: Simulation with Ga \& GaInSn eutectic (miscible set-up) using exaggerated diffusion $D_{\text {fake }}=100 D=$ $1.7 \times 10^{-7} \mathrm{~m}^{2} \mathrm{~s}^{-1} ; J=4 \times 10^{5} \mathrm{Am}^{-2} ; t=31.35 \mathrm{~s}$. The interface shown here is the iso-surface $\rho=\left(\rho_{1}+\rho_{2}\right) / 2$.

Discussion The present work suggests that experiments on the metal pad roll instability can be done with pairs of metals that are liquid at room temperature: $\mathrm{Ga} \& \mathrm{Hg}$ and $\mathrm{Ga} \&$ GaInSn eutectic. The $\mathrm{Ga} \& \mathrm{Hg}$ combination is convenient as immiscibility allows experiments to be run many times. But since the toxicity of $\mathrm{Hg}$ raises regulation issues, the $\mathrm{Ga} \& \mathrm{GaInSn}$ combination is an interesting alternative, although they would be expensive consumables since they would unavoidably be blended after a while. The most important message from our theory is that small set-ups are preferential. Taking $B_{z}=15 \mathrm{mT}$ as a guide and focusing on the $\mathrm{Ga}$ \& $\mathrm{Hg}$ pair, let us compare the following three set-ups
$R=H_{1}=H_{2}=(1,2,5) \mathrm{cm}$. The theory shows that the critical current densities triggering the MPR instability are $J_{c}=(1.80,1.12,0.65) \times 10^{5} \mathrm{Am}^{-2}$ and the corresponding currents are $I_{c}=(57,140,513) \mathrm{A}$. The values $57 \mathrm{~A}$ and $140 \mathrm{~A}$ are reasonable, but $513 \mathrm{~A}$ may pose serious technical issues. Hence using $\mathrm{Ga} \& \mathrm{Hg}$ may not be practical in set-ups larger than a few $\mathrm{cm}$.

In the supplementary material we provide a suggestive sketch of an experimental set-up and discuss a list of difficulties that can be overcome. Inhomogeneity in the electrical current density and magnetic field can cause electrovortex flows [16, but we estimate that a nearly $100 \%$ inhomogeneity is needed for these electrovortex flows to overpower the metal pad roll flow. Precise electrical boundary conditions on realistic solid electrodes ignored in the model are not so important because the proposed set-up is non-shallow. Capillarity, also ignored in the theoretical model, will have weak effects because the capillary length of the $\mathrm{Ga}-\mathrm{Hg}$ system is as small as $0.07 \mathrm{~cm} \ll R$. Finally, ohmic heating remains weak because metals are good electrical and thermal conductors.

The HPC resources were provided by GENCI-IDRIS (grant 2019-0254) in France. J.-L. G. acknowledges support from University Paris Saclay, the National Science Foundation (NSF DMS 1620058, DMS 1619892), the Air Force Office of Scientific Research, (FA9550-18-1-0397), and the Army Research Office (W911NF-15-1-0517).

[1] N. Urata, K. Mori, and H. Ikeuchi, WAA Translation from: J. Jpn. Inst. Light Met. 26, 30 (1976).

[2] T. Sele, Metallurgical and Materials Transactions B 8, 613 (1977).

[3] A. Sneyd, Journal of Fluid Mechanics 156, 223 (1985).

[4] A. Sneyd and A. Wang, Journal of Fluid Mechanics 263, 343 (1994).

[5] V. Bojarevics and M. Romerio, European Journal of Mechanics B/ Fluids 13, 33 (1994).

[6] P. Davidson and R. Lindsay, Journal of Fluid Mechanics 362, 273 (1998).

[7] A. Pedchenko, S. Molokov, J. Priede, A. Lukyanov, and P. Thomas, Europhysics Letters 88, 24001 (2009).

[8] W. Herreman, C. Nore, J.-L. Guermond, L. Cappanera, N. Weber, and G. Horstmann, Journal of Fluid Mechanics 878, 598 (2019).

[9] M. Assael, I. Armyra, J. Brillo, S. Stankus, J. Wu, and W. Wakeham, Journal of Physical and Chemical Reference Data 41, 033101 (2012).

[10] T. Iida and R. Guthrie, The thermophysical properties of metallic liquids (Oxford University Press, Oxford, 2015).

[11] Y. Plevachuk, V. Sklyarchuk, S. Eckert, G. Gerbeth, and R. Novakovic, Journal of Chemical \& Engineering Data 59, 757 (2014).

[12] J.-L. Guermond, R. Laguerre, J. Léorat, and C. Nore, J. Comput. Phys. 221, 349 (2007).

[13] J.-L. Guermond, R. Laguerre, J. Léorat, and C. Nore, J. 
Comput. Phys. 228, 2739 (2009).

[14] L. Cappanera, J.-L. Guermond, W. Herreman, and C. Nore, International Journal for Numerical Methods in Fluids 86, 541 (2018).

[15] W. Herreman, S. Bénard, C. Nore, P. Personnettaz, L. Cappanera, and J.-L. Guermond, Phys. Rev. Fluids
5, $074501(2020)$.

[16] V. Bojarevics, J. A. Freibergs, E. I. Shilova, and E. V. Shcherbinin, Electrically Induced Vortical Flows (Kluwer Academic Publishers, 1989). 